\title{
High numerical aperture holographic microscopy reconstruction with extended $z$ range
}

\author{
N. Verrier ${ }^{a, b}$, D. Donnarumma ${ }^{a}$, G. Tessier ${ }^{c, d}$ and M. Gross ${ }^{a 1}$ \\ 1 a Laboratoire Charles Coulomb - UMR 5221 CNRS-UM2 Université \\ Montpellier Place Eugène Bataillon 34095 Montpellier, France \\ ${ }^{b}$ Laboratoire Hubert Curien - UMR 5516-CNRS-Université Jean Monnet- \\ 18 Rue du Professeur Benoît Lauras 42000 Saint-Etienne, France \\ c Wavefront Engineering Microscopy Group, Neurophotonics Laboratory, \\ CNRS UMR 8250, University Paris Descartes, Sorbonne Paris Cité, 75006 Paris, France \\ d ESPCI ParisTech, PSL Research University, CNRS, \\ Institut Langevin, 1, Rue Jussieu, F-75005 Paris, France
}

\begin{abstract}
An holographic microscopy reconstruction method compatible with high numerical aperture microscope objective (MO) up to $\mathrm{NA}=1.4$ is proposed. After off axis and reference field curvature corrections, and after selection of the +1 grating order holographic image, a phase mask that transforms the optical elements of the holographic setup into an afocal device is applied in the camera plane. The reconstruction is then made by the angular spectrum method. The field is first propagated in the image half space from the camera to the afocal image of the MO optimal plane (plane for which MO has been designed) by using a quadratic kernel. The field is then propagated from the MO optimal plane to the object with the exact kernel. Calibration of the reconstruction is made by imaging a calibrated object like an USAF resolution target for different positions along $z$. Once the calibration is done, the reconstruction can be made with an object located in any plane $z$. The reconstruction method has been validated experimentally with an USAF target imaged with a $\mathrm{NA}=1.4$ microscope objective. Near-optimal resolution is obtained over an extended range $( \pm 50 \mu \mathrm{m})$ of $z$ locations.
\end{abstract}

PACS numbers: 090.1995, 100.3010, 110.0180

N. Verrier, D. Donnarumma, G. Tessier and M. Gross. Appl. Opt. 54, 9540-9747 (2015) http://dx.doi.org/10.1364/A0.54.009540

\section{INTRODUCTION}

In Digital Holography a CCD or CMOS sensor camera records the interference pattern of the object field wavefront with a known coherent reference beam. This digital hologram is then used to numerically reconstruct the image of the object by propagating back the measured object field wavefront from the hologram to the objet [1]. Many reconstruction methods have been proposed for holographic direct imaging, i.e. without microscope objective (MO) 1 [5], and for holographic microscopy (i.e. with MO) [6-11]. In most methods, the holographic reconstruction with MO is made by reconstructing the image of the object enlarged by the microscope objective and not from the object itself. Then the reconstruction is similar to that made in free space. Nevertheless the microscope objective changes the phase of the reconstructed image that must be therefore compensated. Usually this phase compensation is done by adding a lens digital mask in the camera plane [8].

Few methods have been proposed for performing the reconstruction with a large numerical aperture microscope objective. Coulomb et al. [11] proposed to add, in the camera plane, additional Zernike phase corrections that are adjusted to optimize the resolution of the reconstruction. These additional phase corrections are only valid for an object located in the plane where the adjustment was made. In other words when the object is moved along $z$, the phase corrections must be recalculated.

In this paper, we propose a reconstruction method that can be used with a high numerical aperture microscope objective over an extended $z$ range. This method allows to propagate the hologram (i.e. the optical field) in the object half-space from the image of the camera to the MO optimal plane (plane in which MO aberrations are minimal), and then from the MO optimal plane to the object. The calibration, which is independent of the position of the object, consists in determining the position of the optimal plane and calculating the field in that plane. The proposed reconstruction method and the calibration procedure are validated by a test experiment realized imaging an USAF target by using a high numerical aperture $(\mathrm{NA}=1.4)$ microscope objective.

\section{PRINCIPLES OF RECONSTRUCTION WITH A LARGE APERTURE MICROSCOPE OBJECTIVE}

Reconstruction with a high numerical aperture oil immersion objective (MO), is illustrated in Fig. 1. The beam splitter BS2 is angularly tilted to operate in off axis recording geometry.

To get the best possible resolution in conventional imaging (not holographic), the object (an USAF target for instance) and the camera must be located in the object and image optimal planes $\mathrm{P}^{\prime}$ and $\mathrm{P}$ of the microscope objective MO. This means that the objective MO 


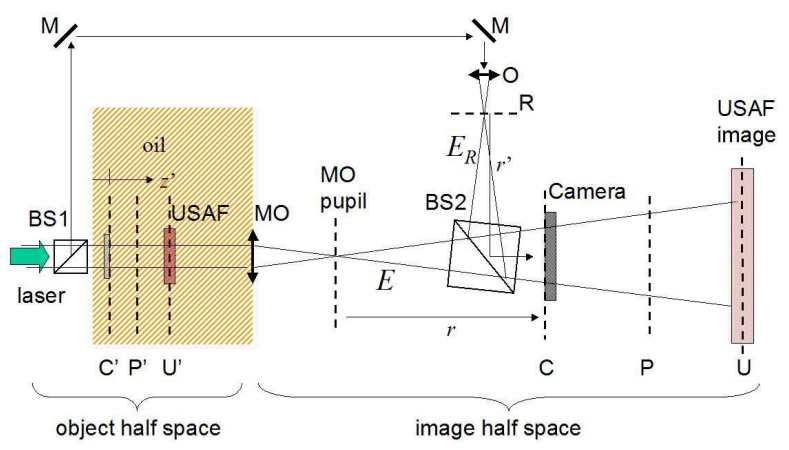

FIG. 1. Typical holographic microscopy setup. BS1, BS2: beam splitters; M: mirror. $E$ and $E_{R}$ : signal and reference optical complex fields; MO: microscope oil objective; USAF: USAF target located in plane U' that is imaged in plane $\mathrm{U}$ by MO; C: camera plane; C': plane of the image of the camera made by $\mathrm{MO}$; $\mathrm{P}^{\prime}$ : optimal $\mathrm{MO}$ object plane; $\mathrm{P}$ : optimal $\mathrm{MO}$ image plane; O: short focal lens objective; $r$ : MO pupil to camera distance; $r^{\prime}$ : radius of curvature of the reference beam in the camera plane C. Note that BS2 is angularly tilted in order to perform off axis holography. Moreover, the reference beam is a spherical wave whose origin is point $R$. $R$ is at infinite if the reference is a plane wave.

has been designed to image an object located in plane $\mathrm{P}^{\text {' }}$ into plane $\mathrm{P}$. The camera planes (C and $\left.\mathrm{C}^{\prime}\right)$, the object planes ( $\mathrm{U}$ and $\left.\mathrm{U}^{\prime}\right)$ and the optimal planes $\left(\mathrm{P}\right.$ and $\left.\mathrm{P}^{\prime}\right)$ must thus coincide $\left(\mathrm{U}=\mathrm{P}=\mathrm{C}\right.$ and $\left.\mathrm{U}^{\prime}=\mathrm{P}^{\prime}=\mathrm{C}^{\prime}\right)$.

If the imaging is done by digital holography there are less constraints. To obtain the best resolution with simple reconstruction that involves quadratic phase propagation kernel, it is sufficient that the object is in the optimal plane $\mathrm{P}^{\prime}$. Thus the condition is $\mathrm{U}=\mathrm{P}$ and $\mathrm{U}^{\prime}=\mathrm{P}$ '. Indeed, the camera can be located in a plane $\mathrm{C}$ that is different from the optimal image plane $\mathrm{U}=\mathrm{P}$, as it is still possible to propagate the field $E$, from the camera plane $\mathrm{C}$ to the plane $\mathrm{U}=\mathrm{P}$ of the image of the object. Since the propagation occurs in free space and since the angles are small, the holographic reconstruction maintains the optimal resolution with quadratic kernel reconstruction.

The proposed reconstruction involves two steps:

1. Field reconstruction in the optimal plane, with quadratic kernel and correction of the phase, to obtain the field in plane P', with the correct amplitude and phase.

2. Field propagation from the optimal plane $\mathrm{P}^{\text {' to the }}$ plane of the object U'. The calculation should be done with the exact propagation kernel, because the propagation takes place in the object half space with angles that can be large.

The reconstruction must be preceded by a calibration procedure to determine:

- The location of the optimal plane P',
- The phase corrections to be applied to obtain the phase of the field $E$ in the optimal plane P'.

- The imaging magnification $G$ to get the pixel size in the optimal plane P', which is needed to calculate the exact propagation kernel.

\section{EXPERIMENTAL SETUP}

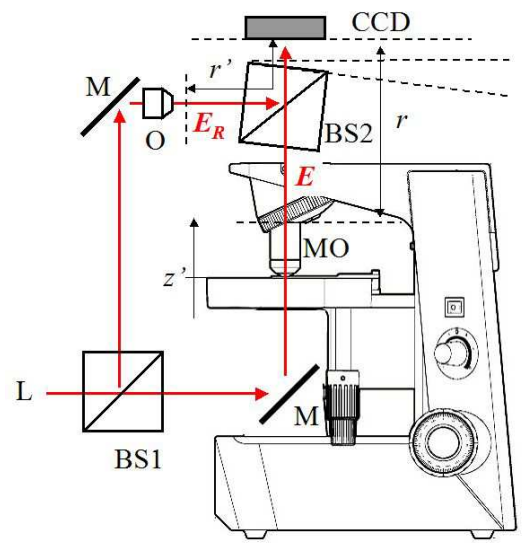

FIG. 2. Experimental setup. $E_{R}, E$ : reference and signal optical fields; L: laser $(785 \mathrm{~nm})$; BS1 and BS2: beam splitters; M: mirror; CCD: CCD camera; O: short focal lens that makes the reference beam divergent; $r$ : MO pupil to camera distance; $r^{\prime}$ : radius of curvature of the reference beam in the camera plane C. $z^{\prime}$ : coordinate of the USAF target plane; MO: microscope objective that image the USAF target.

To illustrate the reconstruction procedure and to perform calibration, an experimental test has been performed by using the Fig. 2 holographic setup.

This setup uses a commercial upright microscope (Olympus CX41) that has been modified; the microscope condenser has been removed and the white light illumination has been replaced by laser illumination. The main laser beam $L$ (Sanyo DL-7140-201: wavelength $\lambda=785$ $\mathrm{nm}$, power $50 \mathrm{~mW}$ for $95 \mathrm{~mA}$ of current) is split by the beam splitter (BS1) into an illumination beam and a reference beam. The object, an U.S. Air Force (USAF) target, is imaged by the microscope objective MO (Nikon: oil, $\mathrm{NA}=1.4, \times 60,160 \mathrm{~mm}$ ). The object field $E$ interferes with the reference field $E_{R}$, and the interference pattern (i.e. the hologram) is recorded by the CCD camera (PCO Pixelfly $1280 \times 1024$ square pixels of size $\Delta x=6.7 \mu \mathrm{m})$. To simplify further digital Fast Fourier Transform (FFT) calculation, the $1280 \times 1024$ matrix measured by the camera is cropped into a $1024 \times 1024$ calculation grid. In order to perform off-axis holography the beam splitter BS2 that mixes the signal and reference fields $E$ and $E_{R}$ is angularly tilted. Moreover, the reference beam is made divergent by the short focal lens $\mathrm{O}$ in order to cover the 

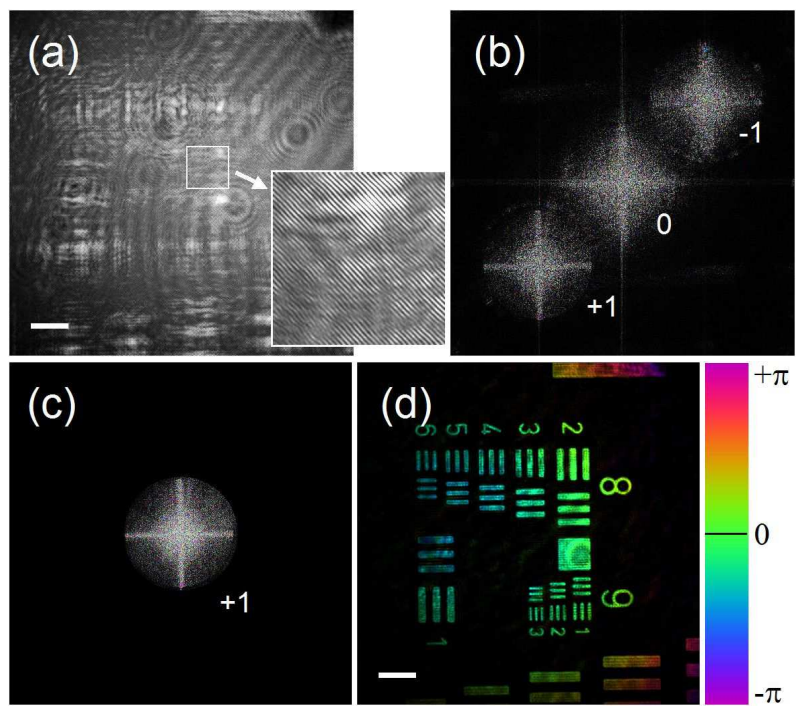

FIG. 3. Holograms $\tilde{H}_{C}$ (a), $\tilde{H}_{2}$ calculated with $d k_{x}=d k_{y}=$ 0 (b), $\tilde{H}_{3}$ calculated with $d k_{x} / \Delta k=255, d k_{y} / \Delta k=-244.52$ and $d k_{\max } / \Delta k=162(\mathrm{c})$ and $H_{3}(\mathrm{~d})$. Holograms are obtained for position $n=1$ (a) and $n=29$ (b,c,d). Arbitrary scale brightness is $\tilde{H}_{C}$ (a), $\left|\tilde{H}_{1}\right|^{2}$ (b), $\left|\tilde{H}_{2}\right|^{2}$ (c) and $\left|H_{2}\right|^{2}$ (d); in (d) color is phase i.e. $\arg \left(H_{2}\right)$. Scale bar is $10 \mu \mathrm{m}(\mathrm{a}, \mathrm{d})$.

whole camera detector area. The wavefront of the reference field $E_{R}$ is thus spherical in the camera plane. Because the described setup has been built by modifying a commercial microscope, the optical distance $r$ from camera to $\mathrm{MO}$ back focal plane (MO pupil plane) and the radius of curvature $r^{\prime}$ of the reference beam wavefront are not known precisely.

In order to calibrate our setup and to evaluate the performance of the reconstruction procedure, holograms of the USAF target have been recorded for $n=1 \ldots 60$ positions along $z$ i.e. for $z_{n}^{\prime} \simeq z_{0}^{\prime}+n \Delta z^{\prime}$ with $n=1 \ldots 60$ and $\Delta z^{\prime}=2.5 \mu \mathrm{m}$. Holograms are recorded with the target located on both sides of camera plane $\mathrm{C}^{\prime}$ that is taken as the origin of coordinates $z^{\prime}=0$. This origin corresponds roughly to position $n=29$. Thus we have $z_{29}^{\prime} \simeq 0$.

\section{HOLOGRAM RECONSTRUCTION IN THE OPTIMAL PLANE P'.}

The hologram recorded in the camera plane $\mathrm{C}$ is:

$$
\begin{aligned}
H_{C} & =\left|E+E_{R}\right|^{2} \\
& =|E|^{2}+\left|E_{R}\right|^{2}+E E_{R}^{*}+E^{*} E_{R}
\end{aligned}
$$

where $E$ and $E_{R}$ are the signal and reference fields in the camera plane C. Figure 3 (a) shows a typical hologram. Due to the off axis configuration, $H_{C}$ exhibits carrier fringes that corresponds to the $E E_{R}^{*}+E^{*} E_{R}$ terms. From $H_{C}$ the hologram $H_{P^{\prime}}$ in the optimal plane $\mathrm{P}^{\prime}$ has been calculated by using the method we have developed

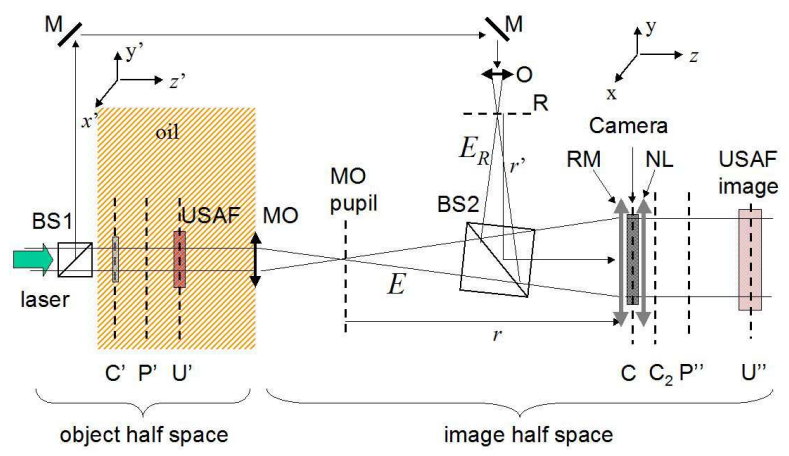

FIG. 4. Reinterpretation of the holographic microscopy setup with the numerical lens NL. USAF: USAF target located in plane U'; MO microscope objective; RM: phase mask located in the camera place $\mathrm{C}$ that acts on the reference field $E_{R}$ to compensate the reference wavefront curvature (focal $\left.r^{\prime}\right)$ and the off axis tilt; NL: numerical lens of focal $r$ located in the camera plane $\mathrm{C}$ that acts on the signal field $E$; MO + NL: afocal optical device that images the USAF target in plane U", and the optimal plane $\mathrm{P}$ ' in plane $\mathrm{P}$ ". The coordinates are $x^{\prime}, y^{\prime}$ and $z^{\prime}$ in the object half spaces, and $x, y$ and $z$ in the object half spaces with respect to $\mathrm{MO}+\mathrm{NL}$. The $z^{\prime}$ and $z$ origins are planes $C^{\prime}$ and $\mathrm{C}$.

in [12]. Here, and in the subsequent text, the term "hologram" indicates a matrix of complex that is calculated during the reconstruction. In most cases, these "holograms" describe the field, or the Fourier transform of the field, in a plane of the object or image half space.

The first reconstruction step is to multiply $H_{C}$ by a complex matrix $C_{R M}$ which describes a phase mask RM applied on the reference field $E_{R}$, whose purpose is to compensate for the wavefront curvature and off axis angle of the reference (see Fig. (4). We have:

$$
\begin{aligned}
& C_{R M}(x, y)=e^{+j k\left(x^{2}+y^{2}\right) / 2 r^{\prime}} e^{j\left(d k_{x} x+d k_{y} y\right)} \\
& H_{1}(x, y)=H_{C}(x, y) C_{R M}(x, y)
\end{aligned}
$$

where $k=2 \pi / \lambda, \lambda$ the wavelength, and $x, y$ the transverse coordinates that are discrete quantities whose step is the pixel size $\Delta x=6.7 \mu \mathrm{m}$. In Eq. 2 the phase factors $e^{+j k\left(x^{2}+y^{2}\right) / 2 r^{\prime}}$ is a lens of focal $r^{\prime}$ that modifies the curvature, and $e^{j\left(d k_{x} x+d k_{y} y\right)}$ a prism that modifies the direction of propagation. The parameters $r^{\prime}, d k_{x}$ and $d k_{y}$ are adjusted so that $E_{R}$ becomes flat field and propagates on axis, i.e. so that the product $C_{R M}(x, y) E_{R}^{*}(x, y)$ does not vary with $x$ and $y$. The +1 grating order terms of $H_{1}$ (i.e. $E C_{R M} E_{R}^{*}$ ) is then proportional to the field $E$ in the camera plane $\mathrm{C}$. Note that the wavefront of $E$ is still curved by MO.

The second reconstruction step is to multiply $H_{1}$ by a second matrix $C_{N L}$ describing a numerical lens NL, located in the camera plane, that acts on the object field $E$ to compensate for the $\mathrm{MO}$ induced wavefront curvature.

$$
\begin{aligned}
& C_{N L}(x, y)=e^{-j k\left(x^{2}+y^{2}\right) / 2 r} \\
& H_{2}(x, y)=H_{1}(x, y) C_{N L}(x, y)
\end{aligned}
$$


where $r$ is the focal of NL. Note that the sign \pm of $j$ in the kernels $e^{+j k\left(x^{2}+y^{2}\right) / 2 r^{\prime}}$ and $e^{-j k\left(x^{2}+y^{2}\right) / 2 r}$ of Eq2 and 3 are opposite. Indeed, the first kernel is supposed to act on $E_{R}^{*}$, while the second kernel to act on $E$. The focal $r$ is adjusted so that MO forms with NL an afocal optical device. Note that for a typical microscope objective MO, the image half space focus plane coincides with the MO pupil, and $r$ is equal to the distance between the pupil and the camera, as shown on Fig. 4.

Introducing a numerical lens NL that transforms the optical elements of the holographic setup into an afocal device is one of the key points of the proposed reconstruction method. This afocal device simplify the reconstruction. Indeed, all the planes that are conjugated by an afocal device have the same phase, and are imaged with the same transverse gain $G$. This gain $G$ is equal to the imaging gain from plane $\mathrm{C}$ ' to $\mathrm{C}$ (with or without NL). Moreover, the longitudinal gain $G_{L}$ remains the same and is finite. Thus, all the conjugated planes of the image half space remains at finite distance $z$. In our test in which an oil immersion objective is used, we have that $G_{L}=G^{2} / n_{m}$, where $n_{m}$ is the oil refractive index.

The +1 grating order term of $H_{2}$ (i.e. $C_{R M} E \times$ $\left.C_{R M} E_{R}^{*}\right)$ is proportional to the field $E$ in a plane $\mathrm{C}_{2}$ located just after the afocal device. This +1 term is selected by spatial filtering in the Fourier space. We must thus calculate the Fourier space hologram $\tilde{H}_{2}\left(k_{x}, k_{y}\right)$ :

$$
\begin{aligned}
\tilde{H}_{2}\left(k_{x}, k_{y}\right) & =\operatorname{FFT}\left[H_{2}(x, y)\right] \\
& =\operatorname{FFT}\left[H_{C}(x, y) e^{-j k\left(x^{2}+y^{2}\right) / 2 r^{\prime \prime}} e^{j\left(d k_{x} x+d k_{y} y\right)}\right]
\end{aligned}
$$

where FFT is the 2D discrete Fourier transform, and $1 / r^{\prime \prime}=1 / r-1 / r^{\prime}$. In Eq. 4, $x, y, k_{x}, k_{y}$ are discrete quantities whose step are $\Delta x$ for $x$ and $y$, and $\Delta k=2 \pi /(N \Delta x)$ for $k_{x}$ and $k_{y}$, where $\Delta x=6.7 \mu \mathrm{m}$ is the pixel size, and $N=1024$ the size of the calculation grid.

The hologram $\left|\tilde{H}_{2}\right|^{2}$ calculated without off axis correction (i.e. with $d k_{x}=d k_{y}=0$ ) is displayed on Fig. 3 (b). The three grating order $(+1,0$ and -1$)$ correspond to the three bright zones of Fig. 3 (b). Should be highlighted here that the edge of +1 grating order is sharp, as noticed in [12]. Indeed, since the NL focal length is equal to the camera-pupil distance $r, \tilde{H}_{2}$ is the reconstructed image of the MO pupil, made by the Schnars et al. method [1] that involves one FFT.

It is good to notice that $\tilde{H}_{2}$ depends on $r^{\prime \prime}$ and not on $r$ and $r^{\prime}$. In section VI will be shown that $r^{\prime \prime}$ can be determined by the calibration procedure, while $r$ and $r^{\prime}$ cannot. Thus, using a plane wave reference $\left(r^{\prime}=\infty\right)$ does not simplify our reconstruction, since a spherical reference $\left(r^{\prime} \neq \infty\right)$ yields similar calculations. Note also that a spherical reference with $r^{\prime} \simeq r$ can be advantageous. Indeed, if $r=r^{\prime}$, the MO pupil is located in the lensless Fourier digital holography plane, and $r^{\prime \prime}=\infty$. The reconstruction of the +1 and -1 images of the pupil is then made by FFT without kernel. The +1 and -1 images are then both sharp, and so them can be separated more easily.

The prism parameters $d k_{x}$ and $d k_{y}$ are adjusted in the way that the pupil image is translated into the center of the Fourier space calculation grid, the Fourier space translation being equal to $d k_{x} / \Delta k, d k_{y} / \Delta k$ in pixels Units. A circular crop of the +1 image of the MO pupil is then made. Since the image of the pupil is sharp, the spatial filtering made by the crop is optimal [12]. The holograms $\tilde{H}_{3}$ and $H_{3}$ obtained with this procedure are:

$$
\begin{aligned}
\tilde{H}_{3}\left(k_{x}, k_{y}\right) & =\tilde{H}_{2}\left(k_{x}, k_{y}\right) \text { if } \sqrt{k_{x}^{2}+k_{y}^{2}}<k_{\max } \\
& =0 \quad \text { if not } \\
H_{3}(x, y) & =\mathrm{FFT}^{-1} \tilde{H}_{3}\left(k_{x}, k_{y}\right)
\end{aligned}
$$

In Eq. 5. the radius of the selected zone is $k_{\max } / \Delta k$ in pixel units. The hologram $\left|\tilde{H}_{3}\right|^{2}$ calculated by Eq. 5 with proper off axis correction $\left(d k_{x} / \Delta k=255\right.$ and $\left.d k_{y} / \Delta k=-244.52\right)$ and circular $\operatorname{crop}\left(k_{\max } / \Delta k=162\right)$ is displayed on Fig. 3 (c).

$\mathrm{H}_{3}$ is equal to +1 grating order of $\mathrm{H}_{2}$ and so is proportional to the field $E$ in plane $\mathrm{C}_{2}$, whose phase is the same than in plane C' (that is conjugated with $\mathrm{C}_{2}$ by the afocal device). This point is illustrated in Fig. 3 (d) that shows $\arg \left(H_{3}\right)$ with colors. Since the control experiment is made with plane wave illumination oriented in the optical axis direction, the phase in planes $\mathrm{C}^{\prime}$ and $\mathrm{C}_{2}$ is flat.

The hologram $H_{P^{\prime \prime}}$ in plane $\mathrm{P}$ ", which is the image of the optimal plane $\mathrm{P}^{\prime}$ made by $\mathrm{MO}+\mathrm{NL}$, is then calculated from $H_{3}$ by propagating the field in the image half space from plane C ( or $_{2}$ ) to plane P". Since P" is at finite distance, this propagation is calculated by the angular spectrum method that involves 2 FFTs [2, 3]. This can be resumed by the following equation:

$$
\begin{aligned}
H_{P^{\prime \prime}}\left(x, y, z_{P^{\prime \prime}}\right) & =\mathrm{FFT}^{-1}\left[e^{j\left(k_{x}^{2}+k_{y}^{2}\right) z_{P^{\prime \prime}} / 2 k} \operatorname{FFT}\left(H_{3}(x, y)\right)\right] \\
& =\mathrm{FFT}^{-1}\left[e^{j\left(k_{x}^{2}+k_{y}^{2}\right) z_{P^{\prime \prime}} / 2 k} \tilde{H}_{3}\left(k_{x}, k_{y}\right)\right]
\end{aligned}
$$

Since the origin of the coordinates $z^{\prime}$ and $z$ are in planes $\mathrm{C}^{\prime}$ and $\mathrm{C}$, the reconstruction distance $z_{P^{\prime \prime}}$ from $\mathrm{C}$ to $\mathrm{P}$ " is the coordinate of plane $\mathrm{P}$ ". This distance is also equal to $z_{P^{\prime \prime}}=z_{P^{\prime}}^{\prime} G_{L}$, where $z_{P^{\prime}}^{\prime}$ is the coordinate of the object optimal plane P', because of the afocal device. The quadratic kernel $e^{j\left(k_{x}^{2}+k_{y}^{2}\right) z_{P^{\prime \prime}} / 2 k}$ describes here the propagation in air from $\mathrm{C}$ to $\mathrm{P}$ ". In Eq. 6 like in previous equations (i.e Eq 2, 3, 4 and 5 ) $x, y, k_{x}, k_{y}$ are discrete quantities whose steps are $\Delta x$ for $x$ and $y$, and $\Delta k$ for $k_{x}$ and $k_{y}$. The quadratic kernel of Eq. 6 can be replaced by the exact kernel $e^{j k_{z} z}$ with $k_{z}=\sqrt{k^{2}-k_{x}^{2}-k_{y}^{2}}$, but since $k_{x}, k_{y}<N \Delta k \ll k$, the two kernel are equivalent.

Note that here the optimal plane P" (and not P) must be taked in account, because this plane is conjugate with P' by the afocal device and so it has the same phase. Note also that the optimal planes P of Fig. 1 and P" of Fig. 4 are very different. To illustrate this point, imagine to have a microscope objective designed for infinite distance correction. Plane $\mathrm{P}$ is thus located at $\infty$. The numerical 
lens NL located in plane $\mathrm{C}$ plays then the role of a tube lens of focal $r$ that moves the optimal plane from $\mathrm{P}$ (at $\infty)$ to $\mathrm{P} "$, whose coordinate is $z_{P^{\prime \prime}}=\left|\mathrm{C} \mathrm{P}^{\prime \prime}\right|=r$.

Because of the afocal device, the hologram (or the field) $H_{P^{\prime \prime}}$ is the exact image of the hologram $H_{P^{\prime}}$ in optimal plane $\mathrm{P}$ ' (having the same amplitude and the same phase) but, due to the $\mathrm{MO}+\mathrm{NL}$ gain $G$, it will be:

$$
\begin{aligned}
& H_{P^{\prime}}\left(x^{\prime}, y^{\prime}, z_{P^{\prime}}^{\prime}\right)=H_{P^{\prime \prime}}\left(x, y, z_{P^{\prime \prime}}\right) \\
& \tilde{H}_{P^{\prime}}\left(k_{x}^{\prime}, k_{y}^{\prime}, z_{P^{\prime}}^{\prime}\right)=H_{P^{\prime \prime}}\left(k_{x}, k_{y}, z_{P^{\prime \prime}}\right)
\end{aligned}
$$

with $x^{\prime}=x / G, y^{\prime}=y / G, k_{x}^{\prime}=G k_{x}$ and $k_{y}^{\prime}=G k_{y}$. Similarly, the hologram $H_{3}$ in plane $\mathrm{C}\left(\right.$ or $\left.\mathrm{C}_{2}\right)$ is the exact image of the hologram $H_{C^{\prime}}$ in the camera image plane C'. It will results also that:

$$
\begin{aligned}
& H_{C^{\prime}}\left(x^{\prime}, y^{\prime}\right)=H_{3}(x, y) \\
& \tilde{H}_{C^{\prime}}\left(k_{x}^{\prime}, k_{y}^{\prime}\right)=\tilde{H}_{3}\left(k_{x}, k_{y}\right)
\end{aligned}
$$

Thus equation 6 can be formally rewritten:

$$
\begin{aligned}
H_{P^{\prime}}\left(x^{\prime}, y^{\prime}, z_{P^{\prime}}^{\prime}\right)= & \mathrm{FFT}^{-1} \\
& {\left[e^{j\left(k_{x}^{\prime 2}+k_{y}^{\prime 2}\right) z_{P^{\prime}}^{\prime} / 2 k_{m}} \tilde{H}_{C}^{\prime}\left(k_{x}^{\prime}, k_{y}^{\prime}\right)\right] }
\end{aligned}
$$

where $k_{m}=n_{m} k$ is the wave vector in oil. In Eq9, $x^{\prime}, y^{\prime}, k_{x}^{\prime}$ and $k_{y}^{\prime}$ are discrete quantities whose steps are $\Delta x / G$ and $G \Delta k$. Although Eq9 seems to describe the propagation of the field from $\mathrm{C}^{\prime}$ to $\mathrm{P}$ ' in oil; actually it describes the field propagation from $\mathrm{C}$ to $\mathrm{P}$ " in air. Thus, the quadratic kernel $e^{j\left({k_{x}^{\prime}}^{2}+{k_{y}^{\prime}}^{2}\right) z_{P^{\prime}}^{\prime} / 2 k_{m}}$ cannot be replaced by the exact kernel in Eq. 9.

\section{RECONSTRUCTION OF AN OBJECT LOCATED OUTSIDE THE OPTIMAL PLANE: U' $\neq \mathbf{P}^{\prime}$.}

The hologram $H_{U}$, in the USAF plane U' can be obtained from $H_{P}$, by propagating the field in the object half space from plane $\mathrm{P}^{\prime}$ to plane $U^{\prime}$. Here again, the propagation can be calculated by the angular spectrum method [2, 3] that involves 2 FFTs. Nevertheless, since the numerical aperture NA can be large $(\mathrm{NA}=1.4$ in the test experiment), we must use the exact propagation kernel $e^{-j k_{z}^{\prime} z^{\prime}}$, and not the quadratic one $e^{j\left({k_{x}^{\prime}}^{2}+k_{y}^{\prime 2}\right) z^{\prime} / 2 k_{m}}$. We get thus:

$$
\begin{aligned}
H_{U^{\prime}}\left(x^{\prime}, y^{\prime}, z^{\prime}\right)= & \mathrm{FFT}^{-1} \\
& {\left[e^{j k_{z}^{\prime}\left(z^{\prime}-z_{P^{\prime}}^{\prime}\right)} \operatorname{FFT}\left[H_{P^{\prime}}\left(x^{\prime}, y^{\prime}, z_{P^{\prime}}^{\prime}\right]\right]\right.}
\end{aligned}
$$

where $\left(z^{\prime}-z_{P^{\prime}}^{\prime}\right)$ is the propagation distance from plane $\mathrm{P}^{\prime}$ to plane $\mathrm{U}^{\prime}$, and $k_{z}^{\prime}$ the $z$ component of the wave vector $\mathbf{k}$ in oil of optical index $n_{m}$. $k_{z}^{\prime}$ is a function of $k_{x}^{\prime}$ and $k_{y}^{\prime}$. We have:

$$
k_{z}^{\prime}=\sqrt{k_{m}^{2}-k_{x}^{\prime 2}-k_{y}^{\prime 2}}
$$

with $k_{m}=n_{m} 2 \pi / \lambda$. In equations [10] and 11, $x^{\prime}, y^{\prime}$ and $k_{x}^{\prime}, k_{y}^{\prime}$ are discrete quantities whose steps are $\Delta x / G$ and $G \Delta k$. We can combine Eqs. (9) and (10) to get:

$$
\begin{aligned}
& H_{U^{\prime}}\left(x^{\prime}, y^{\prime}, z^{\prime}\right)=\mathrm{FFT}^{-1} \\
& {\left[e^{j k_{z}^{\prime}\left(z^{\prime}-z_{P^{\prime}}^{\prime}\right)} e^{j\left(k_{x}^{\prime 2}+k_{y}^{\prime 2}\right) z_{P^{\prime}}^{\prime} / 2 k_{m}} \operatorname{FFT}\left[H_{C^{\prime}}\left(x^{\prime}, y^{\prime}\right)\right]\right]}
\end{aligned}
$$

This equation summarize the reconstruction from $H_{C^{\prime}}$ to $H_{U^{\prime}}$ in a very compact and useful way. This equation is nevertheless difficult to interpret since the two kernels describes two different physical process. The first kernel $e^{j\left(k_{x}^{\prime 2}+k_{y}^{\prime 2}\right) z_{P^{\prime}}^{\prime} / 2 k_{m}}$ is quadratic. It describes the propagation of the hologram in air from C to $\mathrm{P}$ ". Indeed, for the discrete coordinates $k_{x}^{\prime}, k_{y}^{\prime}$ that are used in the calculation, $e^{j\left(k_{x}^{\prime 2}+k_{y}^{\prime 2}\right) z_{P^{\prime}}^{\prime} / 2 k_{m}}$ is exactly equal to the kernel $e^{j\left(k_{x}{ }^{2}+k_{y}{ }^{2}\right) z_{P^{\prime \prime}} / 2 k}$ that describe the C to P" propagation. On the other hand, the second kernel $e^{j k_{z}^{\prime}\left(z^{\prime}-z_{P^{\prime}}^{\prime}\right)}$ describes the propagation in oil from $\mathrm{P}^{\prime}$ to $\mathrm{U}$ '.

Le us summarize the Eq. 12 reconstruction:

- The field is first propagated from the camera image plane C' to the optimal plane $\mathrm{P}^{\prime}$ by using the quadratic kernel $e^{j\left(k_{x}^{\prime 2}+k_{y}^{\prime 2}\right) z_{P^{\prime}}^{\prime} / 2 k_{m}}$, which describes the propagation of the field in the image half space from $\mathrm{C}$ ( or $\mathrm{C}_{2}$ ) to $\mathrm{P}$ ".

- The field is then propagated from the optimal plane $\mathrm{P}^{\prime}$ to the object plane U'. As this propagation is done in the object half-space with a high numerical aperture, we must use the exact kernel $e^{j k_{z}^{\prime}\left(z^{\prime}-z_{P^{\prime}}^{\prime}\right)}$.

\section{CALIBRATION OF THE EXPERIMENTAL SETUP.}

Before performing the reconstruction, it is necessary to calibrate the setup. This calibration consists of the determination of the parameter $r^{\prime \prime}$ of Eq. 4. the prism parameters $d k_{x}, d k_{y}$ of Eq. 2 and 4, the afocal magnification $G$ and the position $z_{P^{\prime \prime}}$ (or $z_{P^{\prime}}^{\prime}$ ) of the optimal plane $\mathrm{P} "$ (or $\mathrm{P}$ ').

The calibration of $G, r^{\prime \prime}$ and $d k_{x}, d k_{y}$ is made by using the afocal idea as follows [12].

- The imaging magnification $G$ is measured by positioning the USAF target in the direct imaging plane $\left(\mathrm{U}^{\prime}=\mathrm{C}^{\prime}\right)$ and by measuring the magnification from plane $\mathrm{U}^{\prime}=\mathrm{C}^{\prime}$ to plane $\mathrm{U}=\mathrm{C}$. In our test experiment, we got $G=74.64$ yielding an pixel size of $\Delta x^{\prime}=89.8 \mathrm{~nm}$ in the object half space. Note that $G$ is not equal to the nominal gain of the our objective $(\times 60)$.

- The parameter $r^{\prime \prime}$ is obtained by adjusting $r^{\prime \prime}$ so that the size of the reconstructed image does not depend on the position $z^{\prime}$ of the USAF target. The magnification is then equal to $G$ for all positions $z^{\prime}$ of the USAF target and in particular for $z^{\prime}=0$ (plane $\mathrm{C}^{\prime}$ to plane $\mathrm{C}$ or $\mathrm{C}_{2}$ ) and $z^{\prime}=z_{P^{\prime}}^{\prime}$ (plane $\mathrm{P}^{\prime}$ to plane $\mathrm{P} ")$. We got $1 / r^{\prime \prime}=-0.58 \mathrm{~m}^{-1}$. 
- The prism parameters $d k_{x}, d k_{y}$ are obtained by adjusting $d k_{x}$ and $d k_{y}$ so that the $x^{\prime}, y^{\prime}$ position of the reconstructed image does not depend on the position $z^{\prime}$ of the USAF target. We got $d k_{x} / \Delta k=255$ and $d k_{y} / \Delta k=-244.52$.
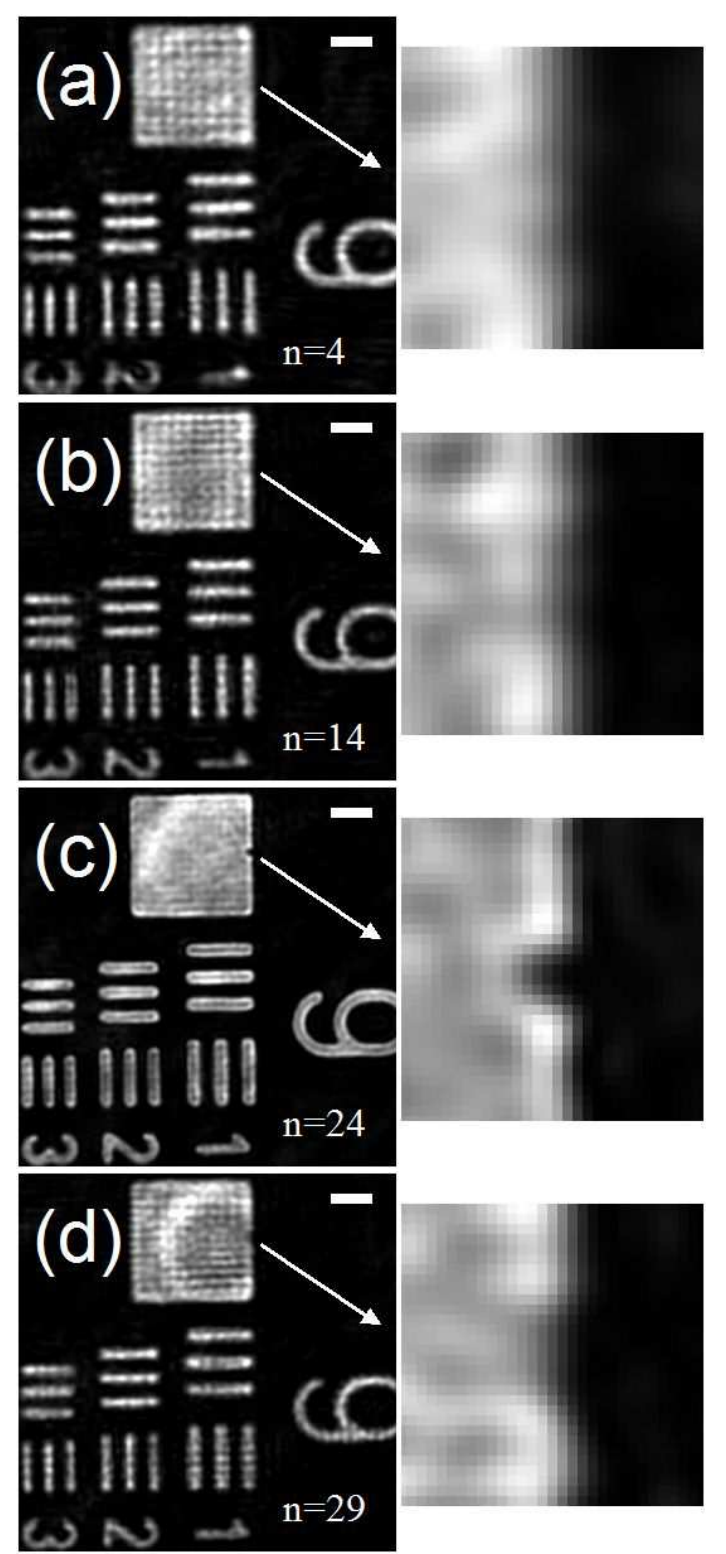

FIG. 5. Zooms $(300 \times 300$ and $30 \times 30$ pixels $)$ of the center of the reconstructed image $\left|H_{U^{\prime}}\right|^{2}(1024 \times 1024$ pixels $)$ of the USAF target obtained for the locations: $n=4$ (a), $n=14$ (b), $n=24$ (c) and $n=29$ (d). Numerical aperture is NA=1.4. $H_{U^{\prime}}$ is calculated by Eq. 12 with $z_{P^{\prime}}^{\prime}=0$ by adjusting $z_{n}^{\prime}$ to minimize $S$. Calculation is made with $z_{n}^{\prime}=-63.69$ (a), -38.54 (b), -13.39 (c) and $-0.86 \mu \mathrm{m}$ (d). Display is made in arbitrary linear scale. Scale bar is $3 \mu \mathrm{m}$, pixel size is $\Delta x^{\prime}=89.8 \mathrm{~nm}$.

The calibration of the location $z^{\prime}{ }_{P}$, of the optimal plane $\mathrm{P}^{\prime}$ is made by calculating the USAF image with a quadratic kernel and by finding the position that gives the best reconstructed image. For all position $n$ of the USAF target, we have thus calculated $H_{U^{\prime}}\left(x^{\prime}, y^{\prime}, z_{n}^{\prime}\right)$ by Eq. 12 with $z_{n}^{\prime}-z_{P^{\prime}}=0$ (i.e.with the quadratic kernel only). For each position $n$, we have adjusted $z_{n}^{\prime}$ so as to obtain the sharpest image $H_{U^{\prime}}\left(x^{\prime}, y^{\prime}, z_{n}^{\prime}\right)$. As the USAF target is an "amplitude object", we have adjusted $z_{n}^{\prime}$ by using the "focus plane detection criterion" of Dubois et al. [13], which minimizes $S$ :

$$
S=\sum_{x^{\prime}, y^{\prime}}\left|H\left(x^{\prime}, y^{\prime}, z_{n}^{\prime}\right)\right|
$$

where $H$ is the hologram that is considered. Here, $H=$ $H_{U^{\prime}}$.

Figure 7 shows zooms $(300 \times 300$ and $30 \times 30$ pixels $)$ of the reconstructed images $(1024 \times 1024$ pixels $)$ obtained after adjustment of $z_{n}^{\prime}$ for positions $n=4$ (a), 14 (b) 24 (c) and 29 (d). The figure illustrates the effect of the USAF position on the quality of the holographic quadratic kernel reconstruction. Position $n=24$ has the best visual resolution and corresponds to the absolute minimum of the criterion $S$. Indeed, the absolute minimum of $S$ is reached for $n=24$ and $z_{24}^{\prime}=-13.39 \mu \mathrm{m}$. Position $n=24$ of the USAF target corresponds thus roughly to the optimal plane (i.e. $\mathrm{U}^{\prime}=\mathrm{P}^{\prime}$ ), with $z_{P^{\prime}}^{\prime} \simeq z_{24}^{\prime}=-13.39 \mu \mathrm{m}$. When the USAF target is located outside the optimal plane (i.e. for $U^{\prime} \neq \mathrm{P}^{\prime}$ ), the optimal resolution cannot be reached and the resolution is lower as seen on Fig. $5(\mathrm{a}, \mathrm{b})$ and (d) that display the best reconstructed images that have been obtained for positions $n=4,14$ and 29 .

By selecting the best position $n, z_{P^{\prime}}^{\prime}$ is measured with an accuracy that is limited by the USAF $z$ displacement step $(2.5 \mu \mathrm{m})$. To avoid this quantization problem, we have determined $z_{P^{\prime}}^{\prime}$ by adjusting both $z^{\prime}-z_{P^{\prime}}^{\prime}$ and $z_{P^{\prime}}^{\prime}$ in Eq. 12 so as to minimize $S$. For position $n=24$, we got $z_{24}^{\prime}-z_{P^{\prime}}^{\prime}=0.8 \mu \mathrm{m}$ and $z_{P^{\prime}}^{\prime}=-14.34 \mu \mathrm{m}$.

We have thus consider, here and in the following, that the exact location of the optimal plane $\mathrm{P}^{\prime}$ is $z_{P^{\prime}}^{\prime}=$ $-14.34 \mu \mathrm{m}$.

\section{RECONSTRUCTION WITH A TUBE LENS AND A MICROSCOPE OBJECTIVE CORRECTED AT INFINITE DISTANCE.}

All the results presented here have been obtained with an objective corrected a finite distance $(150 \mathrm{~mm})$ and without tube lens. There remain nevertheless valid with a tube lens TL and with an objective MO corrected at finite or infinite distance [12]. The tube lens is a long focal length lens located somewhere between the MO pupil and the camera.

Figure 6] shows an example of setup with a tube lens TL located in between the MO pupil and the beam splitter BS2. The tube lens TL modify the optical arrangement, but it is nevertheless possible to find a numerical lens NL that transforms the ensemble of lenses $(\mathrm{MO}+\mathrm{TL}+$ $\mathrm{NL}$ ) into an afocal device. The focuses $r$ and $r^{\prime}$ of both the numerical lens NL and the reference mask RM must 


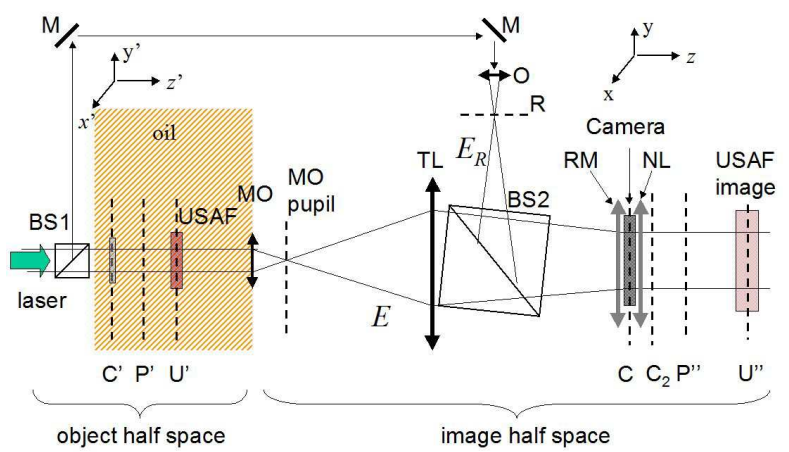

FIG. 6. Holographic microscopy setup with a tube lens TL that is very similar to the Fig 4 setup.

be modified to account for the tube length, but this is done by the calibration procedure, which adjusts $r^{\prime \prime}$ so that the USAF target image keeps the same size for all position $z^{\prime}$. The position $z_{P^{\prime \prime}}$ of the optimal plane and the afocal gain $G$ are modified too, but the calibration procedure, which is based on the afocal character of the ensemble of lens $(\mathrm{MO}+\mathrm{TL}+\mathrm{NL})$ remains the same.

\section{EXPERIMENTAL VALIDATION OF THE USAF RECONSTRUCTION FOR ALL $h$ POSITIONS.}

To validate the proposed method, we have performed the reconstruction for all positions $n=1 \ldots 60$ of the USAF target by calculating $H_{U^{\prime}}^{\prime}$ given by Eq. 12 For each $n$ position, we have calculated $H_{U^{\prime}}^{\prime}$ with $z_{P^{\prime}}^{\prime}=$ $-14.34 \mu \mathrm{m}$ by adjusting $z_{n}^{\prime}$ in order to minimize $S$.

Figure 5 presents the zooms $(300 \times 300$ and $30 \times 30$ pixels) of the center of the USAF images $(1024 \times 1024$ pixels) reconstructed for $n=4,14,24$ and 29. To better visualize the resolution, we have center the $30 \times 30$ pixels zoom on a small defect in the bright square zone of the USAF target (white arrow). Note that the pixels, whose size is $\Delta x^{\prime}=89.8 \mathrm{~nm}$, are visible on the $30 \times 30 \mathrm{zoom}$.

Figures 5 and 7 , allow us to compare the quadratic kernel simplified calculation made in [12] (Fig. 5) with the full two kernels calculation of Eq. 12 (Fig. 7).

- For $n=4$ and $n=14$, the USAF target is shifted by $z_{n}^{\prime}-z_{P^{\prime}}^{\prime} \simeq-50$ and $-25 \mu \mathrm{m}$ with respect to the optimal plane $\mathrm{P}^{\prime}$. The resolutions obtained with the simplified reconstruction are very degraded (Fig 5 (a) et (b) ), while the ones obtained with two kernels are good (Fig:7 (a) et (b)).

- For $n=24$, the USAF target is near the optimal plane $z_{24}^{\prime} \simeq z_{P^{\prime}}^{\prime}$. The full and simplified calculations are mainly made with the quadratic kernel and the images Fig 5 (c) and Fig 7 (c) are nearly identical.

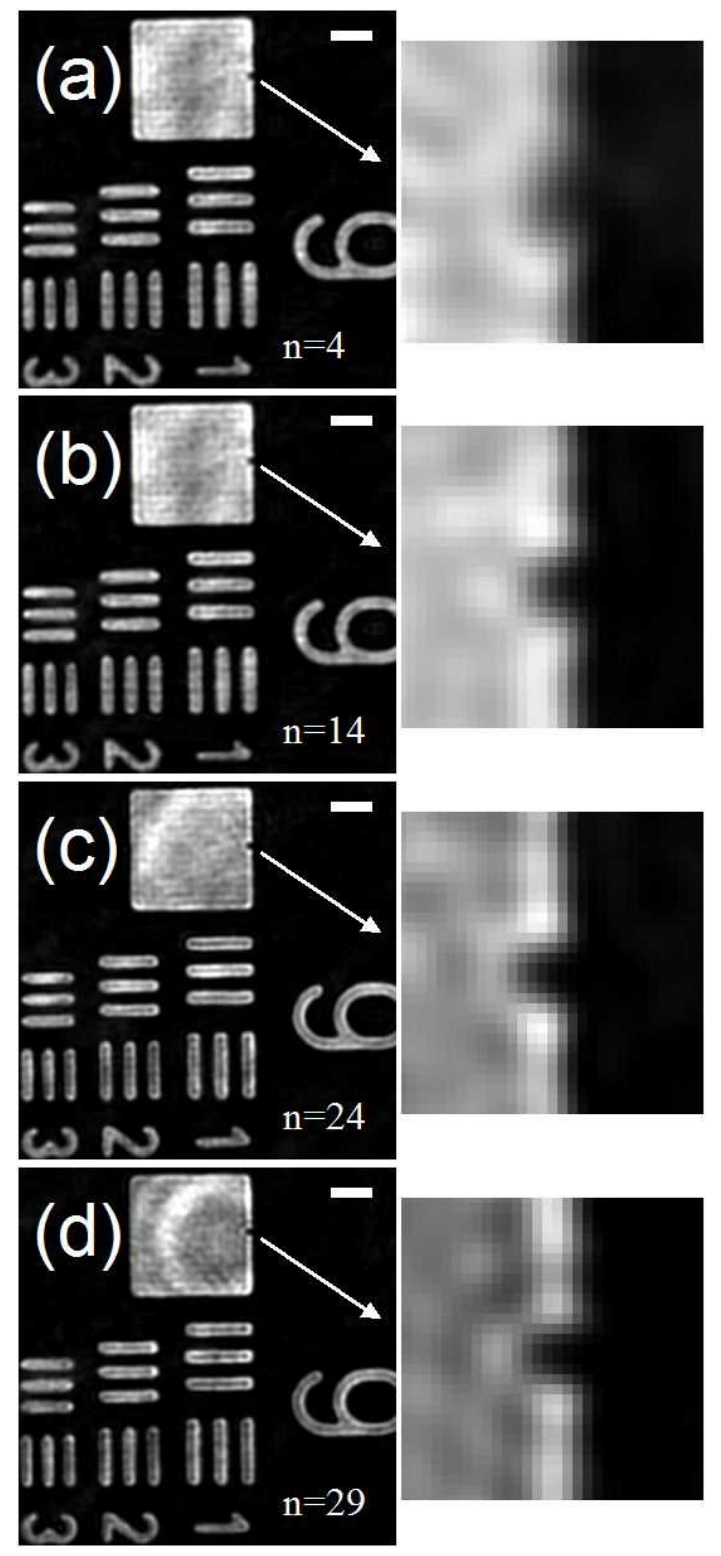

FIG. 7. Zoom $(300 \times 300$ pixels $)$ of the center of the reconstructed image $\left|H_{U^{\prime}}\right|^{2}(1024 \times 1024$ pixels $)$ of the USAF target obtained for the locations: $n=4$ (a), $n=14$ (b), $n=24$ (c) and $n=29$ i.e. (d). Numerical aperture is $\mathrm{NA}=1.4$. $H_{U^{\prime}}$ is calculated with Eq. 12 by adjusting $z_{n}^{\prime}-z_{P^{\prime}}^{\prime}$ and $z_{P^{\prime}}^{\prime}$ to minimize $S$. Calculation is made with $z_{P^{\prime}}^{\prime}=-14.34 \mu \mathrm{m}$ and $z_{n}^{\prime}-z_{P^{\prime}}^{\prime}=-47.69(\mathrm{a}),-23.39(\mathrm{~b}), 0.8$ (c) and $12.84 \mu \mathrm{m}(\mathrm{d})$. Display is made in arbitrary linear scale. Scale bar is $3 \mu \mathrm{m}$, pixel size is $\Delta x^{\prime}=89.8 \mathrm{~nm}$.

- For $n=29$, the USAF target is shifted by $z_{29}^{\prime}-$ $z_{P^{\prime}}^{\prime} \simeq+13 \mu \mathrm{m}$ with respect to $\mathrm{P}^{\prime}$, and close the camera plane $\mathrm{C}^{\prime}$. The simplified reconstruction is thus done nearly without any kernel since $z_{29}^{\prime} \simeq$ 0 . Even in that case, the simplified reconstruction remains very imperfect (Fig 5 (d) ), while the full reconstruction is excellent (Fig:7 (d)). 

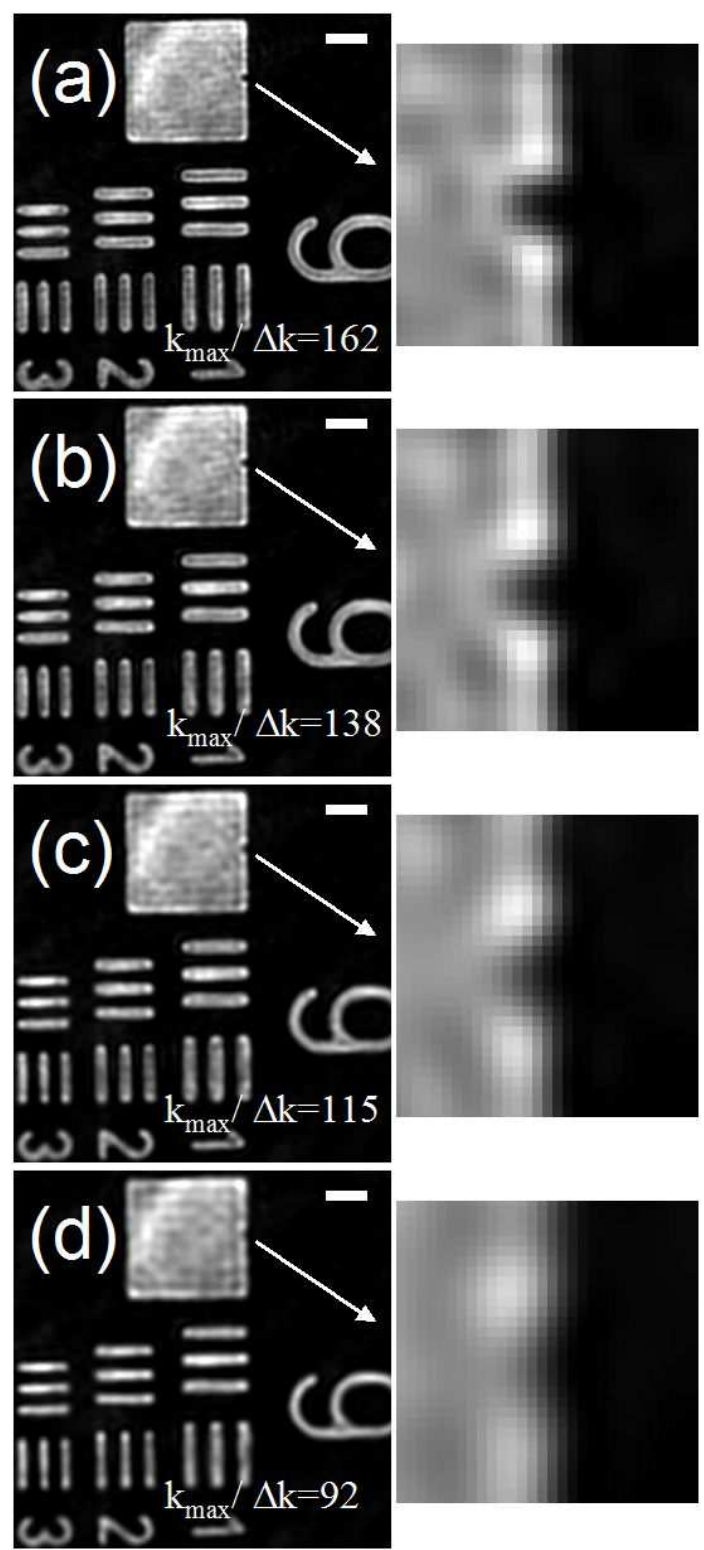

FIG. 8. Zooms $(300 \times 300$ and $30 \times 30$ pixels $)$ of the center of the reconstructed image $\left|H_{U^{\prime}}\right|^{2}(1024 \times 1024$ pixels $)$ of the USAF target obtained for the optimal location $n=24 . \quad H_{U^{\prime}}$ is calculated by Eq. 12 with $z_{P^{\prime}}^{\prime}=-14.34 \mu \mathrm{m}$ and $z_{n}^{\prime}-$ $z_{P^{\prime}}^{\prime}=0.8 \mu \mathrm{m}$ by varying the radius of the selected zone: $k_{\max } / \Delta k=162$ i.e. $\mathrm{NA}=1.4(\mathrm{a}), k_{\max } / \Delta k=138$ i.e. $\mathrm{NA} \simeq$ $1.2(\mathrm{~b}), k_{\max } / \Delta k=115$ i.e. $\mathrm{NA} \simeq 1.0(\mathrm{c})$ and $k_{\max } / \Delta k=92$ i.e. $\mathrm{NA} \simeq 0.8(\mathrm{~d})$. Scale bar is $3 \mu \mathrm{m}$, pixel size is $\Delta x^{\prime}=89.8$ nm.

In order to quantify the resolution we have performed the reconstruction by varying the radius $k_{\max }$ of the cropped zone. By the way, we have modified the equivalent numerical aperture (NA) of microscope objective. To simplify the discussion, we have considered that NA and $k_{\max }$ are roughly proportional. Figure 8 shows the images obtained with the USAF target in position $n=24$ close to the optimal plane $\mathrm{P}^{\prime}$ for $k_{\max } / \Delta k=162$ i.e. $\mathrm{NA}=1.4(\mathrm{a}), k_{\max } / \Delta k=138$ i.e. $\mathrm{NA} \simeq 1.2(\mathrm{~b})$, $k_{\max } / \Delta k=115$ i.e. $\mathrm{NA} \simeq 1.0$ (c) and $k_{\max } / \Delta k=92$ i.e. $\mathrm{NA} \simeq 0.8$ (d). As expected, the resolution decreases with NA. Comparison between Fig:7 and Fig 8 quantifies the resolution degradation observed when $\left|z^{\prime}-z_{P^{\prime}}^{\prime}\right|$ increases.

- For $\left|z^{\prime}-z_{P^{\prime}}^{\prime}\right| \leq 25 \mu \mathrm{m}$, the resolution seen on Fig[7 (b) et (d) is excellent and remains comparable to the resolution obtained for $\mathrm{NA} \simeq 1.2$ on $\mathrm{Fig} 8$ (b).

- For $\left|z^{\prime}-z_{P^{\prime}}^{\prime}\right| \simeq 50 \mu \mathrm{m}$ the resolution seen on Fig 7 (a) deteriorates slightly. It is lower than that obtained for $\mathrm{NA} \simeq 1.2$ on Fig 8 (b), but noticeably better than that the one obtained for $\mathrm{NA} \simeq 1.0$ on Fig 8 (c).

- For $\left|z^{\prime}-z_{P^{\prime}}^{\prime}\right| \leq 5 \mu \mathrm{m}$ the resolution seen on Fig 7 (d) is the same than for $\mathrm{NA}=1.4$ on Fig 8 (c).

Note that the low degradation of the resolution, that is observed on Fig:7, is obtained only if the off-axis tilt parameters $d k_{x}, d k_{y}$ are properly adjusted. To avoid spherical aberration with high NA objective, it is indeed necessary that the reconstruction $z$ axis exactly coincides with microscope objective symmetry axis. By adjusting $d k_{x}, d k_{y}$ so that the $x^{\prime}, y^{\prime}$ position of the reconstructed image do not depend on the $z^{\prime}$ position of the USAF target, we have aligned the $z$ reconstruction axis with the $z$ axis of translation of the object. As our experimental device is constructed by modifying a commercial microscope, the calibration procedure makes the $z$ axis coincident with the MO optical axis, and spherical abberations are minimized.

\section{CONCLUSION}

We have proposed an holographic microscopy reconstruction method compatible with high numerical aperture microscope objective MO. The key idea is to apply in the plane of the camera a phase mask $(\mathrm{RM}+\mathrm{NL})$ that transforms the optical elements of the holographic setup into an afocal device. This mask makes also the standard phase corrections: phase curvature induced by the microscope objective, phase curvature of the reference, and off axis phase.

The reconstruction can be then make in 3 steps:

- The hologram is first propagated in air from the camera plane $\mathrm{C}$ to the image P" of the MO optimal plane P' (in the object half space) by the afocal device. Since P" is at finite distance, the propagation is made by the angular spectrum method. Since propagation is made in the image half space with small angles and large pixels in $x$ and $y$, the calculation can be made with a quadratic kernel.

- The hologram in the MO optimal plane $\mathrm{P}^{\prime}$ is then calculated from the hologram in plane $\mathrm{P}$ " by changing the scale of the coordinates $x, y, k_{x}$ and $k_{y}$. 
- To the end, the hologram is propagated from $\mathrm{P}^{\prime}$ to the object by the angular spectrum method. Since propagation is made in the object half space with large angles and small pixels, this second propagation must be calculated with the exact kernel.

This 3 steps reconstruction can be formally written in 2 steps with a propagation of the hologram from the camera image plane C' to object optimal plane $\mathrm{P}^{\prime}$ with a quadratic kernel, followed by a propagation from $\mathrm{P}^{\text {' to }}$ the object with the exact kernel.

The proposed reconstruction is parameterized by few calibrations parameters that are

- the focal length $r^{\prime \prime}$ of RM+NL phase mask,

- the off axis translation $d k_{x}, d k_{y}$ in Fourier space,

- the microscope enlargement factor $G$ that is needed to change the scale of the coordinates,
- and the location $z_{P^{\prime}}^{\prime}$ of the optimal plane $\mathrm{P}^{\prime}$ in the object half space.

These parameters are easily determined by imaging an object like an USAF target for different positions along $z^{\prime}$. Once the calibration is done, the reconstruction can be made with an object located in any plane $z^{\prime}$.

The reconstruction method has been validated with a USAF target that is imaged with a $\mathrm{NA}=1.4$ microscope objective. With proper calibration, near-optimal resolution over a wide range of sample locations along the $z$ axis can be obtained. We get a resolution better than the one obtained with $\mathrm{NA} \simeq 1.0$ within a range of \pm $50 \mu \mathrm{m}$ in $z$, and better than the one obtained with NA $\simeq 1.2$ for range of $\pm 25 \mu \mathrm{m}$. To get the best results with high NA objective, it is of paramount importance to correctly adjust the reconstruction $z$ axis to make it precisely coincides with the MO optical axis in order to avoid spherical abberation. This can be done easily if the translation of the object made in calibration coincide with the microscope objective optical axis.

We acknowledge ANR Blanc Simi 10 (n 11 BS10 015 02) grant and Labex Numev (convention ANR-10-LABX20) grant for funding.
[1] U. Schnars and W. Jüptner. Direct recording of holograms by a ccd target and numerical reconstruction. Appl. Opt., 33, 179-181, 1994.

[2] F. Le Clerc, L. Collot, and M. Gross. Numerical heterodyne holography with two-dimensional photodetector arrays. Opt. Lett., 25, 716-718, 2000.

[3] L. Yu and M.K. Kim. Wavelength-scanning digital interference holography for tomographic three-dimensional imaging by use of the angular spectrum method. Opt. Lett., 30, 2092-2094, 2005.

[4] F. Zhang, I. Yamaguchi, and L.P. Yaroslavsky. Algorithm for reconstruction of digital holograms with adjustable magnification. Opt. Lett., 29, 1668-1670, 2004.

[5] P. Picart and J. Leval. General theoretical formulation of image formation in digital fresnel holography. J. Opt. Soc. Am. A, 25, 1744-1761, 2008.

[6] P. Ferraro, S. De Nicola, A. Finizio, G. Coppola, S. Grilli, C. Magro, and G. Pierattini. Compensation of the inherent wave front curvature in digital holographic coherent microscopy for quantitative phase-contrast imaging. Appl. Opt., 42, 1938-1946, 2003.

[7] P. Ferraro, S. De Nicola, A. Finizio, G. Pierattini and G. Coppola Recovering image resolution in reconstructing digital off-axis holograms by Fresnel-transform method. Appl. Phys. Lett., 85, 2709-2711, 2004.

[8] F. Montfort, F. Charrière, T. Colomb, E. Cuche, P. Mar- quet, and C. Depeursinge. Purely numerical compensation for microscope objective phase curvature in digital holographic microscopy: influence of digital phase mask position. J. Opt. Soc. Am. A, 23, 2944-2953, 2006.

[9] T. Colomb, F. Montfort, J. Kühn, N. Aspert, E. Cuche, A. Marian, F. Charrière, S. Bourquin, P. Marquet, and C. Depeursinge. Numerical parametric lens for shifting, magnification, and complete aberration compensation in digital holographic microscopy. J. Opt. Soc. Am. A, 23, 3177-3190, 2006.

[10] T. Colomb, J. Kühn, F. Charrière, C. Depeursinge, P. Marquet, and N. Aspert. Total aberrations compensation in digital holographic microscopy with a reference conjugated hologram. Opt. Express, 14, 4300-4306, 2006.

[11] T. Colomb, E. Cuche, F. Charrière, J. Kühn, N. Aspert, F. Montfort, P. Marquet, and C. Depeursinge. Automatic procedure for aberration compensation in digital holographic microscopy and applications to specimen shape compensation. Appl. Opt., 45, 851-863, 2006.

[12] N. Verrier, D. Alexandre, G. Tessier, and M. Gross. Holographic microscopy reconstruction in both object and image half-spaces with an undistorted three-dimensional grid. Appl. Opt., 54, 4672-4677, 2015.

[13] F. Dubois, C. Schockaert, N. Callens, and C. Yourassowsky. Focus plane detection criteria in digital holography microscopy by amplitude analysis. Opt. Express, 14, 5895-5908, 2006. 